\title{
Benefit corporations: ¿̇hacia una primacía renovada del accionista?
}

\author{
Benefit Corporations: Towards a Renewed \\ Shareholder Primacy?
}

\section{Benefit Corporations: Para uma primazia renovada do acionista?}

\author{
Rolando Roncancio Rachid ${ }^{\star}$ \\ Diógenes Lagos Cortés ${ }^{\star *}$ \\ Sebastián Cortés Mejia ${ }^{\star \star *}$
}

Fecha de recibido: 8 de junio de 2017

Fecha de aprobado: 14 de diciembre de 2017

Doi: http://dx.doi.org/10.12804/revistas.urosario.edu.co/empresa/a.5834

Para citar: Roncancio Rachid, R., Lagos Cortés, D., \&, Cortés Mejía, S. (2018). Benefit corporations: ¿¿Hacia una primacía renovada del accionista? Universidad \& Empresa, 20135), 143-168. Doi: http://dx.doi. org/10.12804/revistas.urosario.edu.co/empresa/a.5834

\section{RESUMEN}

El objetivo de este trabajo es reflexionar acerca de si las benefit corporations (BC) conducen a una nueva primacía basada en la búsqueda del bienestar de todos los grupos de interés. Para ello, se comparan las BC con la norma de la primacía del accionista desde tres aspectos: propiedad y control, priorización de intereses y deberes fiduciarios. Se concluye

* Ph.D. y Magíster en Gobierno y Cultura de las Organizaciones, Instituto de Empresa y Humanismo, Universidad de Navarra (España). Magíster en Dirección de Empresas, Inalde Business School, Universidad de La Sabana (Colombia). Profesor del Departamento de Dirección General del Inalde Business School, Universidad de La Sabana. Correo electrónico: rolando.roncancio@inalde.edu.co

** Doctor en Ciencias de la Administración, Universidad Nacional de La Plata (Argentina). Magíster en Ingeniería Industrial, Universidad Industrial de Santander (Colombia). Investigador en Inalde Business School, Universidad de La Sabana. Correo electrónico: diogenes.lagos@inalde.edu.co

*** Estudiante de doctorado en Management, Iowa State University (Estados Unidos). Administrador de Negocios Internacionales, Universidad de La Sabana (Colombia). Ingeniero Industrial, Universidad de La Sabana (Colombia). Correo electrónico: sebas3cm@iastate.edu 
que en las BC: a) la propiedad y el control se mantiene en los accionistas; (b) la maximización del valor considera el bienestar de todos los grupos de interés, y (c) los deberes fiduciarios se amplían para considerar los posibles efectos en los demás grupos de interés, aunque esto no genera ninguna obligación hacia ellos.

Palabras clave: Benefit corporation, primacía del accionista, gobierno corporativo, gestión de los grupos de interés.

\section{ABSTRACT}

The purpose of this study is to ponder how the benefit corporations (BC) lead to a new organizational primacy by perusing wellness for all the stakeholders. Therefore, BC are compared with the norm of the shareholder primacy, from three perspectives: property and control, prioritized interests, and fiduciary duties. It is concluded that in the $\mathrm{BC}: a)$ property and control remain in shareholders who hold the power to create or finish BC's commitments, and the mechanisms to guarantee the fulfillment of the purpose of the organization; $b$ ) maximization of the value in $\mathrm{BC}$ is different because it pursuits the wellbeing of all the stakeholders, nevertheless, it is pointed out that this goal is difficult to reach; c) although fiduciary duties are enlarged to consider possible effects of $\mathrm{BC}$ on all stakeholders, this does not imply an obligation of the organization with any of these groups, in practice, only the discretion of managers who keep their loyalty and care towards shareholders is enlarged.

Keywords: Benefit corporation, shareholder primacy, corporate governance, stakeholder's management.

\section{RESUMO}

O objetivo deste trabalho é refletir acerca de se as Benefit Corporations (BC) conduzem a uma nova primazia baseada na busca do bem-estar de todos os grupos de interesse. Para isso, comparam-se as $\mathrm{BC}$ com a norma da primazia do acionista desde três aspetos: propriedade e o controle, priorização de interesses, e deveres fiduciários. Conclui-se que nas BC: a) a propriedade e o controle se mantém nos acionistas; (b) a maximização do valor considera o bem-estar de todos os grupos de interesses; e (c) os deveres fiduciários se ampliam para considerar os possíveis efeitos nos demais grupos de interesse, no entanto, isto não gera nenhuma obrigação para eles.

Palavras-chave: Benefit corporation, primazia do acionista, governo corporativo, gestão dos grupos de interesse. 


\section{INTRODUCCIÓN}

La estructura del derecho corporativo en Estados Unidos garantiza que las empresas busquen el beneficio de sus accionistas (Smith, 1998), quienes cuentan para ello con mecanismos (elección de directores, aprobación de operaciones relevantes, presentación de reclamaciones) para ejercer el control y exigir que la junta directiva y la alta dirección den prioridad a sus intereses frente a los de otros grupos de interés (Millon, 1993). Este enfoque, centrado en el accionista, es conocido como la norma de la primacía del accionista y es el modelo corporativo y legal predominante en las organizaciones actuales.

Aunque existe consenso en que la norma de la primacía del accionista capta con mayor precisión la personalidad y el propósito de la empresa (Bainbridge, 2003; Chen \& Hanson, 2004), el debate iniciado en los años treinta por los profesores Berle (1931) y Dodd (1932) continúa atrayendo la atención en el ámbito empresarial y académico. Este debate separa a quienes consideran que el propósito único o principal de la empresa es maximizar la utilidad de sus accionistas (shareholder primacy), de quienes consideran que la empresa debe beneficiar a todos los grupos de in- terés (stakeholder theory) (Allen, 1992; Bulter \& McChesney, 1999).

La teoría de los stakeholders y de diferentes ramas relacionadas han recibido recientemente mayor atención en este debate. Esta búsqueda por el bienestar de todos los grupos de interés ha generado una mayor conciencia del impacto negativo que producen las empresas en su intento por mejorar la calidad de vida (Blount \& Offei-Danso, 2013). En este sentido, los defensores de la teoría de los stakeholders sugieren que las empresas, dados los impactos que generan con sus actividades, deberían preocuparse más allá de los intereses de sus accionistas.

En las formas híbridas o empresas de doble misión se facilita la búsqueda de un beneficio económico, a la vez que se genera un impacto positivo directo en la sociedad y el medioambiente (McDonnell, 2014). Este tipo de empresas han asumido que la búsqueda del beneficio para sus accionistas no es su único objetivo, pues estos buscan un balance entre objetivos sociales y económicos. Sin embargo, algunos autores consideran que el derecho corporativo en los Estados Unidos limita la búsqueda de fines sociales en las empresas, cuando estos no se traducen en un aumento de utilidades para los accionistas (Kerr, 2007). En 
este proceso, las formas híbridas de empresa se enfrentan a una serie de limitaciones que afectan su desempeño. Por ejemplo, la dificultad para captar inversores con motivaciones sociales dado que la ley les impide (en el caso de las organizaciones sin ánimo de lucro) o limita (en el caso de las organizaciones híbridas) la repartición de utilidades (Nass, 2013).

Recientemente la legislación de los Estados Unidos ha creado una nueva forma corporativa para promover las formas híbridas de empresa, las Benefit Corporations (BC). ${ }^{1}$ Las $\mathrm{BC}$ responden a la necesidad de articular objetivos sociales con objetivos económicos, generalmente considerados opuestos en el derecho corporativo (Clark \& Vranka, 2013), y consideran como elemento fundamental para la toma de decisiones el posible impacto entre los diferentes grupos de interés (B Lab, 2016). En este contexto, se plantean las siguientes preguntas: ¿las BC están cambiando el paradigma de la primacía del accionista hacia uno nuevo basado en la primacía de los demás grupos de interés?, o simplemente, ¿son una reafirmación de la primacía del accionista? Este trabajo responde a estas cuestiones. Para ello, se contrastan las características de la norma de la primacía del accionista con las características de las BC desde tres aspectos: (a) ¿en quién reside la propiedad y el control de la empresa? (b) ¿cómo se priorizan los intereses de los grupos de interés? (c) ¿a quiénes se extienden los deberes fiduciarios? y ¿qué amplitud tienen estos deberes fiduciarios?

En resumen, se argumenta que en las BC: a) la propiedad y el control continua en las manos de los accionistas quienes detentan las facultades para crear o terminar dicha figura, además de los mecanismos para garantizar el cumplimiento del propósito de la $\mathrm{BC}$; (b) la conceptualización de la maximización del valor es diferente en las $\mathrm{BC}$, ya que estas empresas buscan el bienestar de todos los grupos de interés; no obstante, se advierte que esta situación es compleja de alcanzar en la práctica; y (c) aunque los deberes fiduciarios se amplían para considerar los posibles efectos de las acciones de la $\mathrm{BC}$ en los demás grupos de interés, esta ampliación

1 Las benefit corporations son una nueva forma corporativa creada en los Estados Unidos para promover las formas híbridas de empresa; su uso se ha extendido a otros países como Italia, Francia, Bélgica y Reino Unido. Una traducción al español sería "Corporaciones de Beneficios", aunque, dada la poca difusión del término en español, en este trabajo hemos preferido usar el término en inglés. A enero de 2017, 31 estados habían aprobado leyes que dan vida a las BC, mientras que siete estados más se encontraban en el proceso de aprobación. Iniciativas similares están siendo desarrolladas en otros países como Italia, Francia, Bélgica y Reino Unido. 
no genera ninguna obligación jurídica, ni acciones derivativas a favor de estos grupos. En la práctica se amplía la discrecionalidad de los directivos para la toma de decisiones. No obstante, se mantiene la obligación de lealtad y cuidado hacia los intereses de los accionistas por parte de los directivos.

El resto de este documento se estructura como sigue. Posterior a esta introducción se presenta la metodología; a continuación, se identifican las características de la norma de la primacía del accionista y de las $\mathrm{BC}$. Seguido a esto, se discute acerca de si las BC abren el camino hacia una nueva primacía que contempla a todos los grupos de interés. Este trabajo finaliza con las conclusiones y algunas consideraciones para posibles investigaciones futuras.

\section{PLANTEAMIENTO DEL} PROBLEMA: LAS BC CONDUCEN HACIA UNA PRIMACÍA DE LOS DEMÁS GRUPOS DE INTERÉS

Aunque algunos autores consideran que el marco jurídico actual de los Estados Unidos no impone la primacía del accionista como una camisa de fuerza, es cierto que cuando los directivos toman decisiones que implican una reducción en el beneficio de los accionistas, se exponen a posibles demandas al no cumplir con la regla de la maximización de los beneficios para el accionista. En este contexto, desde lo que parece ser un punto de inflexión que busca hacer de los negocios una cuestión rentable económicamente, pero que a su vez genere un beneficio público importante (Orts, 1992), se han desarrollado un amplio número de organizaciones que no pueden funcionar bajo la lógica de la maximización de utilidades (Mickels, 2009). No obstante, estas organizaciones se enfrentan a una serie de limitaciones que pueden afectar su desempeño, por ejemplo, la dificultad para captar inversores con motivaciones sociales dado que la ley les impide ( a las organizaciones sin ánimo de lucro) o limita (a las organizaciones híbridas) la repartición de utilidades (Nass, 2013). Para lidiar con estas dificultades, se han propuesto varias alternativas, entre estas los estatutos de los grupos de interés (Orts, 1992; Oswald,1998; Wallman,1991), normativa según la cual los directivos de las corporaciones pueden considerar intereses de otros grupos (empleados, consumidores, proveedores y comunidad local) en sus decisiones cotidianas (Swanson, 1996).

Aunque los estatutos de los grupos de interés consideran el efecto de las decisiones en otros grupos, algunos autores creen que sigue siendo una visión restringida donde no se 
incluye la comunidad internacional, el medioambiente o consideraciones más amplias de derechos humanos (Mickels, 2009). Las BC buscan superar esta situación, constituyéndose en una respuesta a la necesidad de encontrar nuevas herramientas jurídicas para cumplir de forma efectiva con la doble misión. De cierto modo, las $\mathrm{BC}$ tratan de recuperar lo que al inicio de la historia se exigía como un requisito esencial para la existencia de las organizaciones, el beneficio público intencionado, obligatorio y directo, y en este sentido, se comprometen a maximizar los beneficios de su operación para todos los grupos de interés (B Lab, 2016).

De esta forma, este trabajo tiene como objetivo reflexionar sobre la posible capacidad que tienen las $\mathrm{BC}$ de cambiar el paradigma de la primacía del accionista hacia uno nuevo basado en la primacía de los demás grupos de interés o si son simplemente una reafirmación de la norma de la primacía del accionista. Para ello, se identifican y se contrastan las características principales de las BC y de la norma de la primacía del accionista.

\section{ASPECTOS DISTINTIVOS DE LA PRIMACÍA DEL ACCIONISTA Y LAS BC}

\subsection{Características de la primacía del accionista}

La norma de la primacía del accionista tiene su origen en la resolución de conflictos entre accionistas mayoritarios y minoritarios, y no entre accionistas y otros grupos de interés (Smith, 1998). Sin embargo, su consolidación se fue dando a medida que los tribunales obligaban a los directores corporativos a dar prioridad al interés de los accionistas (no solo los mayoritarios) frente a los intereses de los demás grupos de interés (Smith, 1998).

De acuerdo con Smith (1998), aunque la primacía del accionista ha tenido innumerables formulaciones, la referencia más citada proviene del caso Dodge contra Ford Motor $\mathrm{Co},{ }^{2}$ donde se determinó que:

Una corporación empresarial se organiza y lleva a cabo principalmente para el beneficio de los accionistas. Los poderes de los directores han de emplearse para ese fin. La discrecionalidad de los directores es ejercida

2 Mediante una acción judicial emprendida por los hermanos Dodge, en ese momento propietarios del $10 \%$ de las acciones de Ford Motor Company, se pretendía que Henry Ford (accionista mayoritario y controlador de la compañía) permitiera que se distribuyeran beneficios especiales. El señor Ford basó su decisión en que el dinero se reinvertiría para crear más empleo, ofrecer precios más bajos, subir salarios y beneficiar a la sociedad en general, asegurando que le parecía que la compañía estaba siendo demasiado rentable, y por eso no se repartiría ese dividendo especial (Dodge v. Ford Motor Co, 1919). 
en la elección de los medios para alcanzar ese fin, y no se extiende a un cambio en el fin en sí mismo, a la reducción de las utilidades, o para la no distribución de las utilidades entre los accionistas con el objetivo de destinarlos a otros fines (Dodge contra Ford Motor Co, 1919, p. 684).

La norma de la primacía del accionista puede resumirse en cuatro aspectos: a) propiedad y control, el accionista es propietario de la empresa, por lo tanto, tiene el control formal, decidiendo el propósito y políticas fundamentales; b) maximización de valor, se entiende como maximización de valor la priorización del beneficio del accionista; c) deberes fiduciarios, estos se restringen al accionista, y d) discrecionalidad directiva, existen normas que protegen la actuación de los directivos. A continuación, se desarrollan cada uno de estos aspectos.

\subsection{Propiedad y control}

Aunque los accionistas son quienes proporcionan el capital para la creación y operación de las empresas, el éxito de las mismas no depende exclusivamente de ellos. Por el contrario, es el resultado de la cooperación y el aporte de múltiples actores (empleados, proveedores, directores corporativos, alta dirección, acreedores y Estado, entre otros) (Blount \& Offei-Danso, 2013). A pesar de esto, en la mayoría de las empresas los accionistas tienen el derecho para ejercer el control y disponer de las utilidades (Hansmann, 1996); esto debido a que desde la perspectiva tradicional (primacía del accionista) son considerados como los propietarios de las empresas.

Convencionalmente, el término propietarios en una empresa se usa para designar a "aquellas personas que comparten dos derechos formales: el derecho a controlar la empresa y el derecho de apropiarse de los beneficios residuales de la empresa" (Hansmann, 1988, p. 269). De acuerdo con esto, el primer derecho de un propietario es controlar su empresa, pero ¿por qué es de esta forma? Desde la primacía del accionista se sugiere que si los accionistas son los propietarios de la empresa, a ellos corresponde darle el propósito (decidiendo las políticas fundamentales) y gobernarla de acuerdo con sus intereses (Matheson \& Olson, 1992).

De acuerdo con la teoría contractualista (Jensen \& Meckling, 1976; Kraakman, 2009), los accionistas no tienen ningún estatus privilegiado respecto de los otros contratantes de la sociedad, esto porque, al igual que los demás, aportan algo a cambio de un beneficio para ellos. Desde esta perspectiva, son proveedores de capital, como otros grupos de interés lo son de materias primas o de 
trabajo; sin embargo, la posición de los accionistas se distingue de la de los otros grupos de interés porque soportan un riesgo residual que los demás no asumen. Los accionistas son los últimos en recibir su beneficio, después de que los demás actores en la empresa lo hayan hecho. Este hecho hace evidente que deban detentar el control de la sociedad (Jensen \& Meckling, 1976).

Easterbrook \& Fischel (1983) manifiestan que, si bien es cierto que el derecho de control se podría repartir de manera igual entre todos los participantes, carecería de lógica económica el hecho de que no hubiera una proporción entre la naturaleza y el alcance del interés, y el correlativo derecho a la toma de decisiones. Por ejemplo, los acreedores o directivos no necesariamente tienen alineado su interés económico al desempeño total de la empresa y, por ello, los contratantes que sí tienen un interés de alcance total no asumirían los riesgos de igual forma. Por esa razón, los beneficiarios residuales (los accionistas), cuyo interés depende de la eficiencia general de la corporación, deben tener el derecho al voto exclusivamente $\mathrm{o}$, al menos, prioritariamente. De este modo, el derecho de control da a los accionistas una medida de certeza de que sus intereses serán considerados en primer lugar cuando los directores cor- porativos tomen decisiones (Blount \& Offei-Danso, 2013).

El segundo derecho de los accionistas es recibir las utilidades, ¿por qué esto es así? La primacía de los accionistas plantea que son ellos quienes proporcionan capital a la empresa a cambio de un retorno de utilidades, es decir, el beneficio económico es el principal objetivo del accionista. Así como un consumidor compra un producto, el proveedor de capital compra las utilidades futuras de la empresa. Dicho de otra manera, la empresa vende al cliente un producto o servicio por un precio y a los accionistas les vende las utilidades futuras, con su riesgo correlativo (Boatright, 2006). En este sentido, otorgar el control a quienes asumen el riesgo residual es la protección más adecuada a su posición contractual, ya que el retorno residual (propio del capital) sería demasiado inseguro si ellos no tuvieran el control para que la firma opere hacia la maximización del beneficio (Boatright, 2006).

En resumen, desde la perspectiva tradicional, los accionistas son los propietarios de la empresa, aunque de un modo diferente a lo encontrado en otros tipos de propiedad; en lugar de tener la propiedad de la empresa como una persona tiene la propiedad de una casa o de un automóvil, los accionistas tienen un 
paquete de derechos que incluyen controlar la empresa y recibir las utilidades (Hansmann, 1996).

\subsection{Maximización de valor}

De acuerdo con Fairfax (2006), la primacía del accionista impide que la empresa se enfoque en actividades que no conducen directamente a la obtención de beneficios económicos en el corto plazo para sus accionistas. En este sentido, Friedman (1953) afirma que, "siempre y cuando el comportamiento de la empresa esté informado por el racional de la maximización de las utilidades para el accionista y sea consistente con este, la empresa prosperará" (p. 22). El planteamiento anterior ha conducido a la amplia aceptación del pensamiento de Friedman (1970) acerca del propósito para el cual se crean las empresas:

hay una y sólo una responsabilidad social de la empresa: usar sus recursos en actividades encaminadas a maximizar sus beneficios, siempre y cuando se mantenga en las reglas del juego, es decir, que actúe dentro de la libre competencia, sin engaño ni fraude (p. 6).

Para Argandoña (2007), esta visión no es arbitraria, ya que la ciencia económica muestra que bajo ciertas condiciones se consigue la máxima eficiencia cuando las empresas maximizan el valor para sus propietarios. Este argumento de eficiencia se apoya también en el planteamiento central del interés residual del accionista y del modelo financiero del mercado (Ireland, 2005), el cual sostiene que el precio actual de mercado de la acción refleja el desempeño de todas las utilidades futuras $\mathrm{y}$, por tanto, el desempeño total de la empresa. De este modo, siendo los accionistas los titulares de un interés residual en la empresa, son ellos los más indicados para gestionar eficientemente el control de la misma, pues su interés específico se sintetiza en el valor de mercado de la acción de la que son tenedores y ese valor es, a su vez, el indicador del desempeño integral de la corporación. De esta manera, maximizar el beneficio del accionista conduce a maximizar el valor de la acción.

Según este enfoque, el sistema centrado en el accionista crearía el escenario más eficiente para la generación de riqueza para todos los grupos de interés (Kiarie, 2006). Sin el derecho de control, los accionistas o proveedores del capital demandarían utilidades más altas para compensar el mayor riesgo de su inversión, esto aumentaría el costo del capital y, en consecuencia, el costo de producción para todos los grupos de interés (Boatright, 2006). Así, la primacía del accionista no solo 
es más eficiente, sino "moralmente justificada porque beneficia mejor el interés de todas los grupos de interés y nace del acuerdo voluntario de todos los participantes en el contrato societario" (Boatright, 2006, p. 116).

\subsection{Deberes fiduciarios}

La separación de la propiedad y el control en las empresas genera problemas de organización debido a que los intereses de los directores corporativos pueden no coincidir con los de los accionistas (Matheson \& Olson, 1992). En este tipo de sociedades la junta directiva desempeña un papel fundamental, ya que detenta facultades para la gestión de los negocios y asuntos de la sociedad, entre ellas: nombrar los representantes legales, tomar decisiones administrativas y financieras e, incluso, reformar los estatutos de la sociedad (Adams, Hermalin, \& Weisbach, 2010). No obstante, los poderes y facultades de los directores corporativos y la alta dirección no son ilimitados; su actuación se encuentra enmarcada por los deberes fiduciarios, cuya base está en la exigencia irrestricta de la buena $\mathrm{fe}^{3}$ de los directores (Reyes, 1996).
Aunque la primacía del accionista se manifiesta en toda la estructura del derecho corporativo, es en los deberes fiduciarios donde encuentra su expresión más directa (Smith, 1998). Desde la perspectiva tradicional, se ve a los accionistas como beneficiarios de los activos, mientras la compañía posee la titularidad de los mismos fiduciariamente (Allen, 1992). Como los accionistas son los propietarios de las acciones, también lo son de una parte de los activos de la empresa (Njoya, 2007); por lo tanto, estos activos deben ser administrados fiduciariamente en su beneficio de la manera más eficiente posible.

La premisa fundamental del derecho corporativo imperante establece que los directores y la alta dirección tienen deberes fiduciarios de cuidado y lealtad para con la empresa y sus accionistas, y no frente a otros grupos de interés (Smith, 1998). En efecto, la primacía del accionista impide que los directores corporativos y la alta dirección favorezcan a otros grupos de interés, al considerar apropiado que las corporaciones maximicen el beneficio de sus accionistas a expensas de estos grupos (Fairfax, 2006).

3 En Colombia, la jurisprudencia constitucional ha definido el principio de buena fe como aquel que exige a los particulares y a las autoridades públicas ajustar sus comportamientos a una conducta honesta, leal y conforme con las actuaciones que podrían esperarse de una "persona correcta (vir bonus)". Así, la buena fe presupone la existencia de relaciones reciprocas con trascendencia jurídica, y se refiere a la "confianza, seguridad y credibilidad que otorga la palabra dada" (Corte Constitucional, 2008). 
Entre los deberes fiduciarios se encuentran el deber de cuidado y el deber de lealtad, el primero impone a los administradores la obligación de manejar los negocios de la sociedad con la diligencia que se esperaría de una persona en el manejo de sus propios negocios, dentro de lo cual se incluye el deber de encontrarse informado respecto de:

las políticas y problemas de la empresa mediante una regular asistencia a las juntas, y poner todo su conocimiento y habilidad para la conducción de los negocios sociales. Deben igualmente ordenar o asumir las investigaciones necesarias cuando descubran alguna irregularidad e implantar los correctivos necesarios con el fin de preservar los activos de la sociedad (Castro de Cifuentes, 1986, p. 125).

Por su parte, el deber de lealtad implica que los administradores actúen a favor del interés de la sociedad y no buscando sus propios intereses a costa de la sociedad. Este deber genera a los administradores:

la obligación de evitar situaciones que causen conflictos de interés; además que la información privilegiada a la que en razón de su cargo, posición, actividad o relación tenga acceso no sea utilizada para un beneficio personal; también que los administradores no se apoderen o aprovechen en su propio beneficio de las oportunidades de negocios (Castro de Cifuentes, 1986, p. 132).

En resumen, los deberes fiduciarios implican no sólo que los directores corporativos y la alta dirección deben promover el interés de los accionistas, sino también que no deben ponerse en una posición en la que sus propios intereses puedan entrar en conflicto con los intereses de los accionistas(Smith \& Rönnegard, 2016).

2.5. Discrecionalidad de los directivos

El desarrollo de toda actividad económica implica un riesgo de ganancia o pérdida para la empresa como efecto directo o indirecto de las decisiones adoptadas; por lo tanto, es normal que los directores corporativos y la alta dirección asuman riesgos en el ejercicio de la administración de la empresa. Sin embargo, la jurisprudencia establece que si las decisiones son tomadas a) de buena fe, b) de una forma informada, c) bien intencionada para los mejores intereses de la empresa, y d) sin tener un interés personal en el objeto de esa decisión, los directores corporativos no serán objeto de cuestionamientos o sanciones. Esta presunción se conoce como la regla de la discrecionalidad de los negocios, que permite a los administradores (cuando sus decisiones satisfacen dichos requisitos) tomar 
riesgos comerciales sin temor a ser cuestionados por lo que debieron hacer, y también evita la intervención judicial en el manejo de la sociedad (Palmiter, 1996).

De esta forma, cuando se presenta una reclamación o se cuestiona una decisión de los administradores, los tribunales imponen a la parte impugnante la carga de superar la presunción de discrecionalidad de los negocios probando para ello que: a) el administrador tiene un conflicto de interés en una acción o transacción corporativa, b) el administrador se involucra o permite un comportamiento ilegal, c) la acción tomada por el administrador carecía de propósito comercial racional, y d) existe culpa grave.

La mayoría de las legislaciones estatales en los Estados Unidos permiten a las sociedades expedir reformas a sus estatutos para exonerar a los administradores por violación a su deber de cuidado de la responsabilidad personal pecuniaria, siempre y cuando no se trate de: a) incumplimiento a su deber de lealtad, b) actos $u$ omisiones de mala fe que impliquen mala conducta intencional o ilegalidad conocida, c) aprobación de repartos ilegales y d) obtención de beneficio personal impropio; sin embargo, ninguno de los reglamentos legis- lativos afecta la capacidad de los tribunales de otorgar protección judicial, prohibiendo medidas corporativas que violen los principios de cuidado (Palmiter, 1996, p. 74).

\subsection{Características de las BC}

La ley modelo para las BC (en adelante, MBCL por las siglas en inglés de Model Benefit Corporation Legislation) presenta a las $\mathrm{BC}$ como una nueva forma jurídica, que ofrece a emprendedores e inversionistas la oportunidad de crear o invertir en una empresa que, de forma consciente, se compromete a maximizar los beneficios de su operación para todos los grupos de interés (B Lab, 2016). El MBCL permite identificar cinco aspectos distintivos de las BC (Clark \& Babson, 2012): a) propósito, este se enfoca en crear un impacto material positivo en la sociedad y el medioambiente; b) deberes fiduciarios, los deberes de los directores corporativos son extendidos para considerar los intereses de otros grupos de interés, en adición a los intereses financieros de los accionistas; c) discrecionalidad de los directivos, el campo de acción aumenta y se garantiza la protección jurídica ante sus actuaciones; d) garantías para el cumplimiento del propósito, mediante el informe anual de su desempeño social y ambiental, y la posibilidad de exigir 
procedimientos de cumplimiento, $\mathrm{y}$ e) medidas adicionales para garantizar el doble propósito, definen la forma como se crea y se mantiene en el tiempo una BC. A continuación, se desarrollan cada uno de estos aspectos.

\subsection{Propósito}

Para constituirse como una $\mathrm{BC}$, se requiere que la empresa defina un beneficio público general, entendido este como "un impacto positivo significativo en la sociedad y el medioambiente como un todo" (B Lab, 2016, p. 3). Adicional al beneficio público general, las BC pueden definir uno o más beneficios públicos específicos en el marco de los siguientes siete objetivos:

a) proveer a comunidades o personas, de bajos ingresos o marginadas, con productos o servicios para su beneficio; b) generar oportunidades económicas para las personas o comunidades, más allá la creación de puestos de trabajo; c) proteger o restaurar el medioambiente; d) mejorar la salud humana; e) promover el arte, la ciencia o el avance del conocimiento; f) incrementar los flujos de capital hacia entidades que tienen un propósito de beneficio para la sociedad o el medioambiente; y g) conferir algún otro beneficio particular para la sociedad o el medioambiente (B Lab, 2016, p. 4).

\subsection{Deberes fiduciarios}

La valoración de los criterios de decisión en las $\mathrm{BC}$ está regulada legal y estatutariamente. Estos criterios se enmarcan en el propósito de beneficio público general o específico, que caracteriza a las $\mathrm{BC}$. La sección 301, literal a del MCBL, indica que los directores corporativos, en cumplimiento de sus deberes y en busca del mejor interés de la $\mathrm{BC}$, deben considerar los efectos de las decisiones sobre accionistas, empleados (tanto de la BC, como de sus subsidiarias y proveedores), clientes, factores sociales relacionados con la comunidad (donde opera $\mathrm{BC}$, sus subsidiarias y proveedores), medioambiente local y global y la BC misma (B Lab, 2016). De forma adicional, en la sección 301, literal 2ii del MCBL, se indica que las $\mathrm{BC}$ pueden dar prioridad a algún grupo de interés en el cumplimiento del propósito de beneficio público general o específico (B Lab, 2016).

De esta forma, en las $\mathrm{BC}$ los deberes fiduciarios se amplían para considerar en sus decisiones otros grupos de interés diferentes de los accionistas. Dentro de este deber fiduciario ampliado, los directivos deben tener en cuenta: a) los intereses a corto y a largo plazo de la $\mathrm{BC}, \mathrm{b})$ la posibilidad de que estos 
intereses puedan ser logrados, y c) la capacidad de la BC para lograr el objetivo de beneficio público general o específico (B Lab, 2016). No obstante, en la sección 301, literal d, se establece que los directivos no tienen ningún tipo de deber fiduciario hacia las personas que son beneficiarias del propósito de beneficio público general y específico (B Lab, 2016). Además, la sección 305 , literal c, establece limitaciones a las personas que pueden iniciar las acciones de reclamación por incumplimiento de los objetivos de beneficio público, contempladas en los procedimientos de cumplimiento del beneficio (en adelante, BEP por sus siglas en inglés de Benefit Enforcement Proceeding).

\subsection{Discrecionalidad de los directivos}

Los estatutos de las BC establecen específicamente que el hecho de considerar los intereses de otros stakeholders "no debe constituir una violación de las normas generales para los directores, las cuales requieren de buena fe, el cuidado de una persona prudente, $\mathrm{y}$ la consideración de los mejores intereses de la BC" (Clark y Babson, 2012, p. 848). No cumplir con el propósito general o específico de la BC "puede constituir una violación del deber fiduciario y puede dejar a los directores corporativos, a la alta dirección, o a la misma BC sujetos a una demanda por violación de esta obligación" (McDonnell, 2014, p. 34). Para proteger a los directores y a la misma $\mathrm{BC}$, se establece que estos no serán responsables de los resultados obtenidos, al considerar un componente de generación de valor para otros grupos de interés diferentes de los accionistas (Clark \& Babson, 2012); en este sentido, el MBCL establece limitaciones en la responsabilidad, tanto para los directores corporativos como para la misma BC.

En la sección 301, literal c, se establece que los directores no serán responsables por los daños monetarios causados como resultado de cualquier acción u omisión durante sus labores como directores cuando a) este no presenta conflictos de interés, $\mathrm{y}$ b) existe fracaso de la BC al tratar de obtener o crear un beneficio público general o específico (B Lab, 2016). Todo esto, a menos que la acción u omisión que conduce a dichos resultados sea producto de conflictos de intereses, dolo o la violación de la ley por parte del director (B Lab, 2016). De igual forma, en la sección 301, literal b del MBCL, se limita la responsabilidad de las BC, al definir que estas no serán responsables por daños monetarios al tratar de obtener o crear un beneficio público general o específico (B Lab, 2016). 


\subsection{Cumplimiento del propósito}

Dos mecanismos ayudan a mejorar la transparencia de la gestión en las $\mathrm{BC}$, permitiendo vigilar el cumplimiento del propósito definido, los BEP y el informe anual de beneficio (en adelante, ABR por las siglas en inglés de Annual Benefit Report). Un BEP es "cualquier reclamación, acción o procedimiento iniciado para enmendar: (a) la falla de una $\mathrm{BC}$ para tratar de obtener o crear un beneficio público general o un beneficio público específico que haya sido establecido en sus estatutos, o (b) la violación de cualquier obligación, deber, o norma de conducta en virtud de la legislación para las BC" (B Lab, 2016, p. 3).

La sección 305, literal c, establece que un BEP solo podrá ser iniciado y continuado por: a) la $\mathrm{BC}$ directamente; b) una persona o un grupo de personas que al momento de la acción u omisión reclamada posean una participación de al menos el $2 \%$ del total de las acciones de las clases existentes; c) un director corporativo; d) una persona o un grupo de personas que al momento de la acción $u$ omisión reclamada posean una participación de al menos el $5 \%$ del total de las acciones en circulación en una organización de la cual la $\mathrm{BC}$ es una subsidiaria; y e) otras personas que hayan sido especificadas en los estatutos de la BC.

De otra parte, la legislación de las $\mathrm{BC}$ estipula la obligación de presentar un ABR que acoja el estándar de un tercero, entendido este como "un estándar reconocido para definir, informar y evaluar el desempeño corporativo social y ambiental" (B Lab, 2016, p. 2). Este debe ser exhaustivo, fiable, independiente y transparente (Clark \& Babson, 2012); para ello, el ABR debe contener "una descripción de las vías utilizadas para alcanzar el beneficio público estipulado, las circunstancias que hayan dificultado la creación de dicho beneficio, además de una evaluación sobre el desempeño social y medioambiental" (B Lab, 2016, p. 20).

A diferencia de los reportes financieros, donde existen convenciones aceptadas para informar sobre el desempeño financiero, aún no existe una forma estándar para informar sobre el desempeño social y ambiental, por lo tanto, la legislación permite a las $\mathrm{BC}$ seleccionar el estándar que van a utilizar (Clark \& Babson, 2012). Además, estas disponen de diferentes opciones para realizar sus reportes, entre ellas: B Impact Assessment, Global Reporting Initiative (GRI), ISO 2600, UL 
880, Food Alliance and the Sustainable Agriculture Network (SAN), Green Seal Business, People 4 Earth (Wilburn \& Wilburn, 2014).

\subsection{Medidas adicionales para articular y reforzar la doble misión}

Las BC pueden convivir con cualquier otro tipo jurídico de empresa que busque beneficios. Para sumarse a esta figura jurídica, una empresa nueva o existente debe acoger los artículos de la ley modelo de las BC del estado en el que se incorpora la respectiva sociedad. En el MBCL se establece que una mayoría de dos terceras partes de los accionistas debe aprobar decisiones fundamentales como a) acoger o abandonar el estatus de $\mathrm{BC}, \mathrm{y} \mathrm{b}$ ) realizar fusiones, adquisiciones $\mathrm{u}$ otras operaciones fundamentales de tal magnitud que lleven a la finalización práctica del estatus de BC.

Otra disposición destinada a fortalecer el cumplimiento de la doble misión es la creación de veedores internos, como el Benefit Director (BD) y el Benefit Officer (BO), cuyo régimen de responsabilidad es el mismo de los demás administradores. El nombramiento de un BO es opcional en todas las jurisdicciones, a diferencia del $\mathrm{BD}$, que es obligatorio en la mayoría y, en caso de ser nombrado, deberá elaborar el informe anual de beneficio. El BD es una persona independiente de la compañía que es designado por la junta directiva (B Lab, 2016), y que tiene la responsabilidad de presentar un informe anual declarando si la $\mathrm{BC}$ y sus directores han actuado en cumplimiento del beneficio durante el período pertinente. En caso de que el $\mathrm{BD}$ considere que la $\mathrm{BC}$ no ha cumplido con los requisitos de la ley, este debe describir dicha situación (B Lab, 2016).

\section{3. ¿LAS BC SE TRADUCEN EN UNA PRIMACÍA DE LOS GRUPOS DE INTERÉS DIFERENTES A LOS ACCIONISTAS?}

En este apartado, se contrastan las características de la primacía del accionista con las características de las $\mathrm{BC}$, con el objetivo de determinar si estas conducen hacia una nueva primacía que desplaza el interés de los accionistas hacia el interés de los demás grupos de interés. La discusión se desarrolla desde tres interrogantes: ¿dónde reside la propiedad y el control en las BC?, ¿cómo se entiende la maximización del valor en las $\mathrm{BC}$ ? y ¿sobre quién recaen los deberes fiduciarios en las BC? 
3.1. ¿̇En quién reside la propiedad y el control en las $\mathrm{BC}$ ?

Desde la perspectiva tradicional, el accionista es considerado el propietario de la empresa, dado que tiene el derecho de controlarla y de apropiarse de los beneficios residuales (Hansmann, 1988). Sin embargo, algunos autores plantean que la pretensión de que los accionistas son los propietarios de la empresa es empíricamente incorrecta, ya que estos, aunque tienen el control de la empresa, no son los únicos que tienen intereses residuales (Stout, 2002). En la práctica se evidencia que los accionistas son solo uno de los grupos que esperan recibir beneficios residuales. Por ejemplo, los empleados y los directivos esperan aumentos de salario y estabilidad en el trabajo, los tenedores de bonos esperan un entorno favorable para la empresa, donde el riesgo de insolvencia sea menor. De forma similar a lo que sucede en las empresas tradicionales, en las $\mathrm{BC}$ distintas partes mantienen intereses residuales.

En cuanto al control, las $\mathrm{BC}$ contemplan los BEP como un mecanismo para controlar las actuaciones de los directores; sin embargo, estos procedimientos solo pueden ser presentados por la misma $\mathrm{BC}$, los accionistas (con ciertos requerimientos de participación accionaria), los directores y otros grupos de interés (en caso de que se les haya dado prelación en los estatutos de la BC) (B $\mathrm{Lab}, 2016)$. De esta forma, aunque en las BC la toma de decisiones implica considerar los posibles efectos en los grupos de interés, el control se mantiene en los accionistas, quienes eligen y controlan a los directores corporativos (Lan, 2015). La importancia de los accionistas en las BC es tal, que en disposiciones para reforzar la figura jurídica, se considera un régimen de mayorías (dos terceras partes) para adoptar y abandonar el estatus de BC. Del mismo modo, cuando una empresa realice una fusión o intercambio de acciones que dé lugar a la creación o desaparición de una $\mathrm{BC}$, estas operaciones deberán recibir la aprobación de sus accionistas en la misma proporción.

En resumen, afirmar que los accionistas son o no los propietarios es una cuestión discutible. Por ejemplo, desde una mirada puramente conceptual y tradicional, los accionistas de una $\mathrm{BC}$ son los propietarios de la empresa, ya que detentan el control y el derecho de apropiarse de las ganancias; sin embargo, si consideramos que los accionistas no son los únicos actores con intereses residuales, estos no serían los únicos propietarios. De otra parte, en cuanto al control se tiene que en las $\mathrm{BC}$ se modifica la norma de la 
primacía del accionistas a través de la decisión voluntaria de los mismos, no obstante, estos retienen todos los derechos de voto, por lo tanto, el accionista mantiene la primacía con respecto a la creación y el control de la BC (Blount \& OffeiDanso, 2013).

\section{2. ¿Cómo se entiende la maxi- mización de valor en las $B C$ ?}

La norma de la primacía de los accionistas no solo implica que todos los accionistas reciban un trato igualitario; también exige que sus intereses sean preferibles a los de otros stakeholders (Smith, 1998). Por lo tanto, desde la primacía del accionista, la maximización del valor se entiende como maximizar los intereses de los accionistas. Para algunos autores el enfoque en la maximización de valor para los accionistas no debe ser visto como obligatorio en el propósito de la empresa, sino que debe ser entendido como un enfoque por defecto cuando en los estatutos de la empresa no se establece otro (Bainbridge, 2003; Wishnick, 2011).

Esto es diferente en las $\mathrm{BC}$, ya que en sus estatutos se comprometen a maximizar los beneficios de su operación para todos los grupos de interés (B Lab, 2016). Es decir, amplían su propósito más allá de la maximización del valor de los accionistas.
Sin embargo, autores como Brudney y Ferrell (2002), y Fairfax (2006) coinciden en que la retórica de los stakeholders es incompatible con la realidad de las prácticas corporativas $\mathrm{y}$, por lo tanto, esto parece reflejar solo un deseo por parte de las corporaciones para presentar una imagen de buenos ciudadanos que realmente esconde el verdadero objetivo de maximizar beneficio del accionista (Fairfax, 2006).

En este sentido, Lan (2015) sugiere que la legislación de las $\mathrm{BC}$ presenta tres problemas relacionados con las responsabilidades de los directores, que afectan la concepción de la maximización del valor en estas empresas: a) No orientación, aunque desde la teoría se acepta que la maximización del valor en las $\mathrm{BC}$ es entendida como la maximización del bienestar de todos los grupos de interés, esto en la práctica es complicado de alcanzar, por lo tanto, se debe priorizar en alguno o algunos de estos grupos de interés; sin embargo, la legislación de las BC no proporciona ninguna orientación a los directores con respecto a la forma de elegir o priorizar entre los intereses de los diferentes grupos de interés; b) conflicto de intereses ampliado, se pasa de una figura de principal-agente a una de principales-agente. En el primer caso el interés del director debe estar alineado 
con el interés de los accionista, mientras que en el segundo caso lo está con los demás principales o grupos de interés, además con el de los accionistas; (c) interés público no representado, el interés de los grupos diferentes de accionistas no está representado, irónicamente quienes puede hacer que la $\mathrm{BC}$ persigan el bien social son los accionistas, los directores y la BC en sí misma.

Desde una perspectiva diferente, algunos académicos consideran que la primacía del accionista no es la norma, esto sustentado en casos donde los tribunales han permitido que las empresas beneficien a otros grupos de interés por encima de los propios accionistas, como sucede en las contribuciones a obras de caridad (Fairfax, 2006). No obstante, los defensores de la primacía del accionista sugieren que estos casos son consistentes con la norma, ya que si bien el accionista está renunciando a una parte de su beneficio en el corto plazo, esta acción se realiza con la intención de obtener un beneficio mayor para el accionista en el largo plazo (Fairfax, 2006). Esto se logra a través de la mejora de la imagen de la empresa que procede de estas acciones; adicionalmente, dichas acciones pueden ayudar a aumentar la inspiración de los empleados y la lealtad de los clientes, traduciéndose en mayor beneficio económico para los accionistas (Chen \& Hanson, 2004).

De esta forma, se puede afirmar teóricamente que la concepción de valor en las $\mathrm{BC}$ es totalmente diferente a la encontrada en la primacía del accionista. En las BC la maximización del valor se traslada a todos los grupos de interés, mientras que en la primacía del accionista solo se considera a los accionistas como objetivo de la maximización del valor. No obstante, en la práctica es complicado cumplir estos postulados, ya que esto depende exclusivamente de la voluntad de los accionistas, pues las BC mantienen las características de la primacía del accionista, al ser este el que decide el propósito y las políticas fundamentales de la $\mathrm{BC}$, además de mantener el control formal de la misma.

\section{3. ¿A quiénes se extienden los deberes fiduciarios en las $\mathrm{BC}$ ?}

El beneficio público general es esencial en las BC. Estas empresas deben crear "un impacto positivo significativo en la sociedad y el medioambiente como un todo" (B Lab, 2016, p. 3). Esto implica que no existe un deber de cuidado hacia un grupo de interés en particular, por el contrario, este se enmarca en el deber de obediencia al beneficio 
público general. A primera vista, esto parece contrario a lo que sucede en la norma de la primacía del accionista, donde se da prioridad a los intereses de los accionistas por encima de los intereses de otras grupos de interés (Fairfax, 2006).

A juicio de algunos autores, la visión anterior ha sido malentendida. $\mathrm{Si}$ bien es cierto que en las $\mathrm{BC}$ se amplían los deberes fiduciarios de los directivos (estos ya no son la maximización de beneficios para los accionistas, sino también para con la sociedad en general y el medio ambiente). Esto no quiere decir que los directores tengan algún tipo de deber fiduciario hacia las personas que son beneficiarias del propósito de beneficio público general y específico (André, 2015). La responsabilidad de cumplimiento en el propósito se mantiene hacia los accionistas, es decir, si los directores no cumplen con el propósito de la $\mathrm{BC}$, estarían incumpliendo a los accionistas (quienes voluntariamente han decidido buscar ganancias económicas pero de forma social y ambientalmente responsables) y no a los demás grupos de interés. Lo que sucede realmente es que existe mayor discrecionalidad y amplitud (pues los directivos incorporan en sus criterios de decisión el beneficio de todos los grupos de interés) para quienes toman las decisiones en las
$\mathrm{BC}$, no obstante, la primacía sigue siendo del accionista.

Para André (2015), la legislación de las BC amplía la misión de la empresa (maximización de valor es igual a beneficiar a todos los demás grupos de interés) sin aumentar la responsabilidad de la misma. Por ejemplo, en la sección 301 , literal b del MBCL, se establece que las BC no serán responsables por daños monetarios al tratar de obtener o crear un beneficio público general o específico. De igual forma, en la sección 301, literal c, se exonera de responsabilidades a los directores cuando intentan obtener un beneficio público general o especifico.

Aunque en la legislación de las BC se les pide a los directores considerar en sus decisiones a los grupos de interés diferentes a los accionistas (B Lab, 2016), la legislación hace explicito que dichos grupos no pueden castigar a los directores cuando no se cumple con las obligaciones definidas en los estatutos para con ellos (André, 2015). De esta forma, aunque deben servir a los intereses de los grupos de interés diferentes a los accionistas, los directores pueden evadir fácilmente esta responsabilidad, ya que estos grupos de interés no cuentan con herramientas suficientes para garantizar el cumplimiento de dichas 
responsabilidades (Munch, 2012). En este sentido, la legislación define los BEP como los mecanismos para garantizar el cumplimiento del propósito de las BC; no obstante, se excluye a los grupos de interés diferentes de los accionistas, como potenciales participantes de este mecanismo, de tal forma que deja la responsabilidad final del cumplimiento de su propósito en manos de los accionistas en forma de derechos de voto y BEP (Blount \& Offei-Danso, 2013). Del mismo modo, los accionistas son quienes deciden adoptar o abandonar la forma societaria de una $\mathrm{BC}$, ningún otro grupo de interés lo puede hacer por ellos.

Como puede verse, los directores de una $\mathrm{BC}$ tienen un deber fiduciario con sus accionistas y a ellos subordinan todas sus acciones dentro de los límites de la ley. El marco jurídico de referencia de sus actuaciones sigue siendo la maximización de beneficios para los accionistas, solo que en las BC, por voluntad de los accionistas, se pretende esto desde una perspectiva de responsabilidad social y ambiental (Clark \& Babson, 2012). Es decir, se busca que la empresa sea rentable económicamente para sus accionistas, pero que a su vez genere un beneficio público importante (Orts, 1992).

\section{CONCLUSIONES}

En este trabajo se realiza una comparación entre las características de la primacía del accionista y las $\mathrm{BC}$, con el objetivo de identificar si esta forma jurídica conduce a una nueva primacía basada en el beneficio de los demás grupos de interés o si son simplemente una reafirmación de la primacía del accionista. Esta comparación se hace desde tres interrogantes: a) ¿en quién reside la propiedad y el control?, b) ¿cómo se priorizan los intereses de los diferentes grupos de interés? y c) ¿a quién se extienden los deberes fiduciarios?

Respecto a la propiedad se puede decir que en las BC como en las empresas tradicionales, los accionistas son los propietarios, ya que detentan el control y el derecho de apropiarse de las ganancias. Sin embargo, si no consideramos a los accionistas como los únicos con intereses residuales, estos no serían los únicos propietarios, así que la propiedad de la empresa es una cuestión conceptual que depende desde la perspectiva que se analice. En cuanto al control se evidenció que aunque pareciera que en las BC se modifica la norma de la primacía del accionista por decisión voluntaria de estos, los accionistas en la práctica retienen todos los derechos de voto, por lo tanto, su primacía se 
mantiene respecto a la creación y el control de la BC (Blount \& OffeiDanso, 2013).

Respecto a la maximización del valor, encontramos que las $\mathrm{BC}$ no carecen de un propósito económico, solo que este ha sido redefinido. Al igual que todas las empresas, una $\mathrm{BC}$ está destinada a generar un beneficio por sus accionistas, no obstante, esto se consigue desde una perspectiva de responsabilidad social y ambiental (Clark \& Babson, 2012). Aunque se reconoce que la maximización del valor en las BC es entendida como la maximización del bienestar de todos los grupos de interés, en la práctica es necesario escoger o priorizar algunos de estos grupos. Sin embargo, no existen criterios para realizar esta priorización, lo que hace de la maximización de valor en las $\mathrm{BC}$ una cuestión práctica difícil de alcanzar.

Respecto a los deberes fiduciarios encontramos que en las $\mathrm{BC}$ no existe un deber fiduciario hacia los demás grupos de interés, este sigue residiendo en el accionista, solo que es ampliado en cuanto a su propósito (los directores ahora deberán contemplar los posibles efectos de sus acciones en los demás grupos de interés). Esta situación conduce a un conflicto de intereses ampliado, donde se pasa de una figura de principal- agente a una de principales-agente; esto es resuelto en la legislación de las BC limitando la posibilidad para que otros grupos de interés puedan ejercer los mecanismos de control y verificación para el cumplimento del propósito de la $\mathrm{BC}$.

Este trabajo permite concluir de forma general que, aunque los accionistas retienen todos los derechos de voto en la empresa, la primacía del accionista se renueva para ampliar la misión de la compañía, de tal forma que esta asuma una posición más dialogante hacia los demás grupos de interés, lo que permite una mejor conexión entre la empresa y la sociedad.

\section{REFERENCIAS}

Adams, R. B., Hermalin, B. E., \& Weisbach, M. S. (2010). The Role of Boards of Directors in Corporate Governance: A Conceptual Framework and Survey. Journal of Economic Literature, 48(1), 58-107.

Allen, W. T. (1992). Our Schizophrenic Conception of the Business Corporation. Cardozo Law Review, 14, 261-281.

André, R. (2015). Benefit Corporations at a Crossroads: As Lawyers Weigh in, Companies Weigh their Options. Business Horizons, 58(3), 243-252. 
http://doi.org/10.1016/j.bushor.2014.12.002

Argandoña, A. (2007). Responsabilidad social y creación de valor para el accionista: objetivos contrapuestos o complementarios. IESE Occasional Paper, 3(7), 1-13. Bainbridge, S. M. (2003). Director Primacy: The Means and Ends of Corporate Governance. Northwestern University Law Review, 97, 547-606.

B Lab. (2016). Model Benefit Corporation Legislation. Recuperad de http://benefitcorp.net/sites/ default/files/Model Benefit Corp Legislation_4_16.pdf

Berle, A. A. (1932). For whom Corporate Managers are Trustees: A Note. Harvard Law Review, 45(8), 1365-1372.

Blount, J., \& Offei-Danso, K. (2013). Benefit Corporation: A Questionable Solution to a Non-Existent Problem, The. St. Mary's Law Journal, 44(1), 617-670.

Boatright, J. R. (2006). What's Wrong - and What's Rightwith Stakeholder Management. Journal of Private Enterprise, 22(2), 106-130.

Brakman, R. D. (2011). Benefit Corporations-A Sustainable Form of Organization. Wake Forest $L$. Rev., 46, 591.

Brudney, V., \& Ferrell, A. (2002). Corporate Charitable Giving. The University of Chicago Law Review, 1191-1218.
Bulter, H. N., \& McChesney, F. S. (1999). Why They Give at the Office: Shareholder Welfare and Corporate Philanthropy in the Contractual theory of the Corporation. Cornell Law Review, 84(5), 1195-1226.

Castro de Cifuentes, M. (1986). La responsabilidad de los administradores de las sociedades comerciales: enfoques del derecho angloamericano y del derecho colombiano. Revista de Derecho Privado, (1), 123-141.

Chen, R., \& Hanson, J. (2004). The Illusion of Law: The Legitimating Schemas of Modern Policy and Corporate Law. Michigan Law Review, 103(1), 1-149.

Clark, W. H., \& Babson, E. K. (2012). How Benefit Corporations are Redefining the Purpose of Business Corporations. William Mitchell Law Review, 38(2), 817-851.

Clark, W. H., \& Vranka, L. (2013). The Need and Rationale for the Benefit Corporation: Why it is the Legal Form that Best Addresses the Needs of Social Entrepreneurs, Investors, and, Ultimately, the Public. Recuperado de http:// benefitcorp.net/sites/default/files/ Benefit_Corporation_White_Paper.pdf

Corte Constitucional. (2008). SentenciaC-1194/08. Recuperadodehttp: //www.corteconstitucional.gov.co/ relatoria/2008/c-1194-08.htm 
Dodd, E. M. (1932). For Whom Are Corporate Managers Trustees? Harvard Law Review, 45(7), 1145-1163.

Dodge v. Ford Motor Co. (1919). Dodge v. Ford Motor Co., 170 N.W. 668 (Mich. 1919).

Easterbrook, F. H., \& Fischel, D. R. (1983). Voting in Corporate Law. The Journal of Law \& Economics, 26(2), 395-427.

Fairfax, L. (2006). The Rhetoric of Corporate Law: The Impact of Stakeholder Rhetoric on Corporate Norms. Journal of Corporation Law, 31(3), 675-718.

Friedman, M. (1953). The Methodology of Positive Economics. En Essays in Positive Economics (pp. 3-43). Chicago: University of Chicago Press.

Friedman, M. (1970). The Social Responsibility of Business is tolincrease its Profits. The New York Times Magazine. Recuperado de http://umich.edu/ thecore/ doc/Friedman.pdf

Hansmann, H. (1988). Ownership of the Firm. Journal of Law, Economics, \& Organization, 4(2), 267304.

Hansmann, H. (1996). The Ownership of Enterprise. Boston: Harvard University Press.

Ireland, P. (2005). Shareholder Primacy and the Distribution of Wealth. The Modern Law Review, 68(1), 49-81.
Jensen, M. C., \& Meckling, W. H. (1976). Theory of the Firm: Managerial Behavior, Agency Costs and Ownership Structure. Journal of Financial Economics, 3(4), 305-360.

Kerr, J. E. (2007). Sustainability Meets Profitability: The Convenient Truth of How the Business Judgment Rule Protects a Board's Decision to Engage in Social Entrepreneurship. Cardozo Law Review, 29(2), 623-669.

Kiarie, S. (2006). At Crossroads: Shareholder Value, Stakeholder Value and Enlightened Shareholder Value: Which Road Should the United Kingdom Take? International Company and Commercial Law Review, 17(11), 329-343.

Kraakman, R. H. (2009). The Anatomy of Corporate Law: A Comparative and Functional Approach (2 ed.). New York: Oxford University Press.

Lan, G. (2015). Benefit Corporations: A Persisting and Heightened Conflict for Directors. Journal of Law, Business \& Ethics, 21, 113-117.

Matheson, J. H., \& Olson, B. A. (1992). Corporate Law and the Longterm Shareholder Model of Corporate Governance. Minnesota Law Review, 76, 1313-1391.

McDonnell, B. H. (2014). Committing to Doing Good and Doing Well: Fiduciary Duty in Benefit 
Corporations. Fordham Journal of Corporate \& Financial Law, 20(1), 19-72.

Mickels, A. (2009). Beyond Corporate Social Responsibility: Reconciling the Ideals of a ForBenefit Corporation with Director Fiduciary Duties in the US and Europe. Hastings International and Comparative Law Review, 32(1), 271-293.

Millon, D. (1993). Communitarians, Contractarians, and the Crisis in CorporateLaw. WashingtonandLee Law Review, 50(4), 1373-1393.

Munch, S. (2012). Improving the Benefit Corporation: How Traditional Governance Mechanisms can Enhance the Innovative New Business Form. Northwestern Journal of Law \& Social Policy, 7(1), 170-195.

Nass, M. (2013). Viability of Benefit Corporations: An Argument for Greater Transparency and Accountability. Journal of Corporation Law, 39(4), 875-893.

Njoya, W. (2007). Property in Work: The Employment Relationship in the Anglo-American Firm. Aldershot: Ashgate Publishing.

Orts, E. W. (1992). Beyond Shareholders: Interpreting Corporate Constituency Statutes. The George Washington Law Review, 61(1), 14-135.

Oswald, L. J. (1998). Shareholders v. Stakeholders: Evaluating Corporate Constituency Statutes Un- der the Takings Clause. Journal of Corporate Law, 24(1), 1-29.

Palmiter, A. (1996). Deberes fiduciarios de administradores de empresas en los Estados Unidos. En El Derecho Societario Contemporáneo. Primer Congreso Iberoamericano de Derecho Empresarial (pp. 55-87). Bogotá: Departamento de Publicaciones, Cámara de Comercio de Bogotá. Rawhouser, H., Cummings, M., \& Crane, A. (2015). Benefit Corporation Legislation and the Emergence of a Social Hybrid Category. California Management Review, 57(3), 13-35. http://doi. org/10.1525/cmr.2015.57.3.13

Reyes, V. F. H. (1996). Sociedades comerciales en Estados Unidos: introducción comparativa. Bogotá: Editorial Doctrina y Ley.

Smith, D. G. (1998). Shareholder Primacy Norm.The Journal of Corporation Law, 23, 277-323.

Smith, N. C., \& Rönnegard, D. (2016). Shareholder Primacy, Corporate Social Responsibility, and the Role of Business Schools. Journal of Business Ethics, 134(3), 463-478. http:// doi.org/10.1007/s10551-0142427-x

Stout, L. A. (2002). Bad and Not-SoBad Arguments for Shareholder Primacy. Southern California Law Review, 75, 1189-1209.

Swanson, C. B. (1996). The Turn in Takeovers: A Study in Public 
Appeasement and Unstoppable Capitalism. Georgia Law Review, 30(4), 943-1004.

Wallman, S. (1991). The Proper Interpretation of Corporate Constituency Statues and Formulation of Directors' Duties". Stetson Law Review, 21, 163-167.

Wilburn, K., \& Wilburn, R. (2014). The Double Bottom Line: Pro- fit and Social Benefit. Business Horizons, 57(1), 11-20. http://doi.org/10.1016/j.bushor.2013.10.001

Wishnick, D. A. (2011). Corporate Purposes in a Free Enterprise System: A Comment on eBay v. Newmark. The Yale Law Journal, 121, 2405-2419. 